\title{
Evaluation of Ion Beam Behavior in 50W Class RF Ion Thruster
}

\author{
Huong T. T. Nguyen $\mathbb{D}$, Hoai-Duc Vu $\mathbb{D}$, and Jichul Shin $\mathbb{1}$ \\ Department of Aerospace Engineering, University of Ulsan, 44610, Republic of Korea \\ Correspondence should be addressed to Jichul Shin; jshin@ulsan.ac.kr
}

Received 21 April 2021; Revised 29 June 2021; Accepted 25 August 2021; Published 24 September 2021

Academic Editor: Linda L. Vahala

Copyright $\odot 2021$ Huong T. T. Nguyen et al. This is an open access article distributed under the Creative Commons Attribution License, which permits unrestricted use, distribution, and reproduction in any medium, provided the original work is properly cited.

\begin{abstract}
Phenomenological behavior of ion beam acceleration through the grid system in $50 \mathrm{~W}$ class RF ion thruster has been investigated using PIC simulation and evaluated by experimental test using Faraday probe. Beam trajectory for various grid voltages reveals that the metal engine cover of the ion thruster which is needed to seal RF coil around the discharge chamber affects the beam divergence angle. Simulation result shows that the divergence angle increases by $10.52 \%$ mainly because of the larger radial electric field in the presence of the metal engine cover. The divergence angle increases as the accelerator grid voltage increases. The current density distribution measured by the Faraday probe shows a bigger divergence angle with the engine cover installed. For the test cases with mass flow rates from $3 \mathrm{sccm}$ to $4 \mathrm{sccm}$ at the RF power of about $50 \mathrm{~W}$, the current density distribution exhibits the 2 nd peak at the radial position about $4 \mathrm{~cm}$ from the centerline.
\end{abstract}

\section{Introduction}

Electric propulsion is widely used in satellite propulsion system owing to the successive development of Hall thrusters and ion thrusters. In 1884, Hittorf first introduced the radio frequency ion thruster (RIT) in which the ions are produced by an electromagnetic field [1-4]. It has several advantages such as higher efficiency and higher exhaust speed, and hence, higher specific impulse compared to the other similar class microscale plasma thrusters [5-7]. In the design and development of ion thruster, the grid system must be designed and optimized for individual thrusters in that the divergence angle and morphology of the ion beam are important as it directly affects the specific impulse and thrust. It is reported in the previous experimental and theoretical research that the divergent characteristics of the ion beams produced by single and multiaperture accelerator systems are affected by geometric parameters of the grid and operating conditions [8-12]. However, these studies have not analyzed the influence of the metal cover enclosing the engine on the divergence angle of the ion beam. The engine cover is installed to minimize any unnecessary spark from the grids and to protect the RF coil surrounding the engine.
The induced magnetic field in RF ion thruster is very small and hence Lorentz force mostly depends on the electric field that plays a role in the movement of the particles when passing through the grid aperture. Potential distribution through the grid system affects the ion perveance and plume shape. To evaluate this effect, we adopt PIC simulation $[13,14]$ which is widely used to understand the behavior of the ion beam passing through the grid system of an RF ion thruster for the purpose of determining the divergence angle of the ion beam. The model solves electric field and ions dynamics in the overall domain which is $2 \mathrm{D}$-axisymmetric and includes grids. In order to confirm the simulation result, the ion current density in the radial direction was measured by the Faraday probe system.

\section{Working Principle of a Radio Frequency (RF) Ion Thruster}

Schematic drawing of the gridded radio frequency (RF) ion thruster used in this study is shown in Figure 1. The ions are produced in a conical-shaped discharge chamber by the oscillating electric field which is excited by $13.56 \mathrm{MHz} \mathrm{RF}$ power and heats the electrons to ionize the propellant gas 


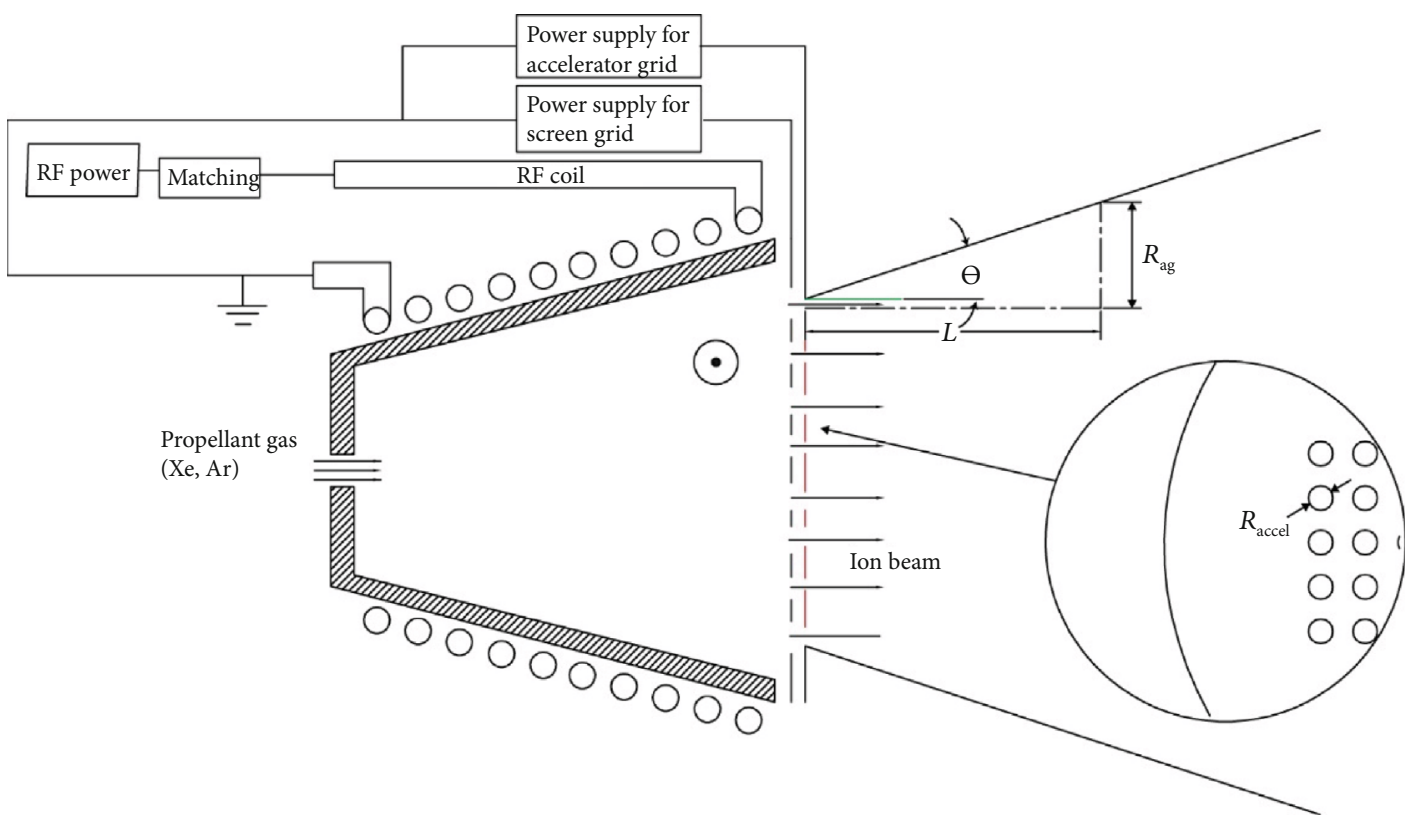

Figure 1: Schematic drawing of the RF ion thruster.

(Xe) [15]. The produced ions are accelerated by the electric field across a two-grid system (screen grid and accelerator grid). Acceleration of the produced ions through the grid system strongly depends on the potential distribution where Child-Langmuir sheath forms near the grid holes and affects the concentration and parallelism of an ion beam. The current density of the ion beam $\left(J_{B}\right)$ through the sheath can be calculated by

$$
J_{B=} \frac{\pi \varepsilon_{0}}{9}\left(\frac{2 巴}{m_{i}}\right)^{1 / 2}\left(\frac{d_{s}}{l_{e}}\right)^{2} V_{T}^{3 / 2},
$$

where $V_{T}$ is total accelerating voltage between the grids, $\mathbb{d}_{s}$ is hole diameter the screen grid, $l_{e}$ is effective acceleration length, $\varepsilon_{0}$ is free space permittivity, and $m_{i}$ is ion mass [12]. Then, the ion perveance per hole becomes

$$
\frac{J_{\mathrm{B}}}{V_{\mathrm{T}}^{3 / 2}}\left(\frac{l_{\mathrm{e}}}{d_{\mathrm{s}}}\right)^{2}=\frac{\pi}{9} \varepsilon_{0}\left(\frac{2 e}{m_{\mathrm{i}}}\right)^{1 / 2},
$$

from which the optimal grid geometries and voltages are chosen to achieve the best perveance. However, this perveance does not account for the beam divergence outside the grid system where the charge distribution can be modified by the electric field formed outside the accelerator grid.

\section{PIC Simulation of the Ion Beam}

The acceleration process of the ions and the divergence phenomenon of the ion beam through the grid system were investigated by PIC simulation featured in OverViz plasma simulation suite [16]. Numerical simulation helps to visualize the ion beam trajectory and provides the ion density distribution from which the divergence angle of the ion beam can be determined [17, 18]. Figure 2 shows two different computational domains with corresponding boundary conditions. Figure 2(a) consists of only two grids, while Figure 2(b) includes the engine cover placed to investigate the effect of the engine cover on the divergence of the ion beam. The self-consistent electrostatic potential is solved using Poisson's equation for charged species.

$$
\vec{\nabla} \cdot\left(\varepsilon_{r} \vec{\nabla} \phi\right)=-\frac{\rho_{c}}{\varepsilon_{0}}
$$

Voltage at the screen grid is set constant at $1500 \mathrm{~V}$, and the accelerator grid voltages are varied from $-350 \mathrm{~V}$ to $-250 \mathrm{~V}$ to investigate the effect of total acceleration voltage on the beam divergence. The metal engine cover is grounded. Singly ionized xenon is launched at uniform density over the gray area marked on Figure 2 and is accelerated through the grid system. Ion motions under the calculated electric field are solved using PIC method in which the ion kinetics are calculated by Newton's law [19]. The divergence angle is calculated by [12].

$$
\theta=\tan ^{-1}\left[\frac{\left(R_{a}-R_{B}\right)}{L}\right],
$$

where $L$ is the distance from the accelerator grid to the right exit plane, $R_{B}$ is the radius of the ion beam at the accelerator grid, and $R_{a}$ is the radial extent of ion density distribution at the right exit plane where the propellant gas leaves the computational domain as shown in Figure 2. Grid parameters used in the simulation is listed in Table 1. 


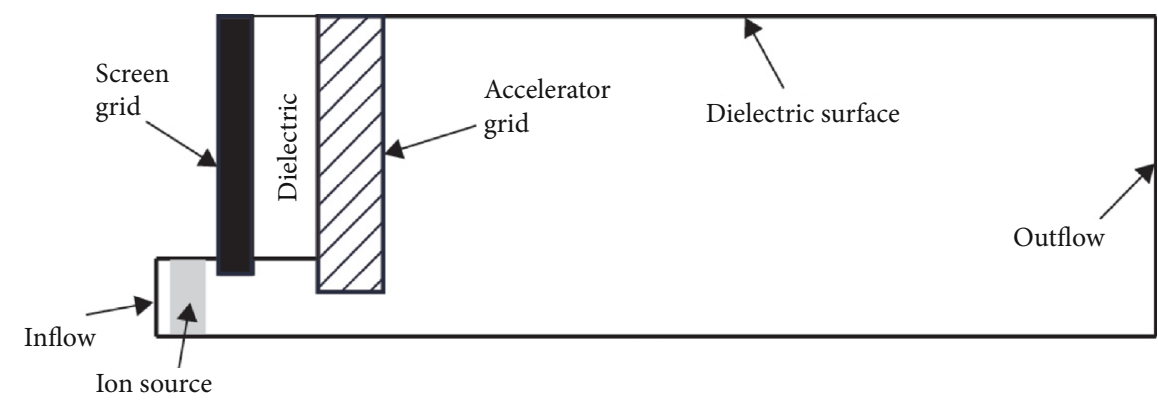

(a)

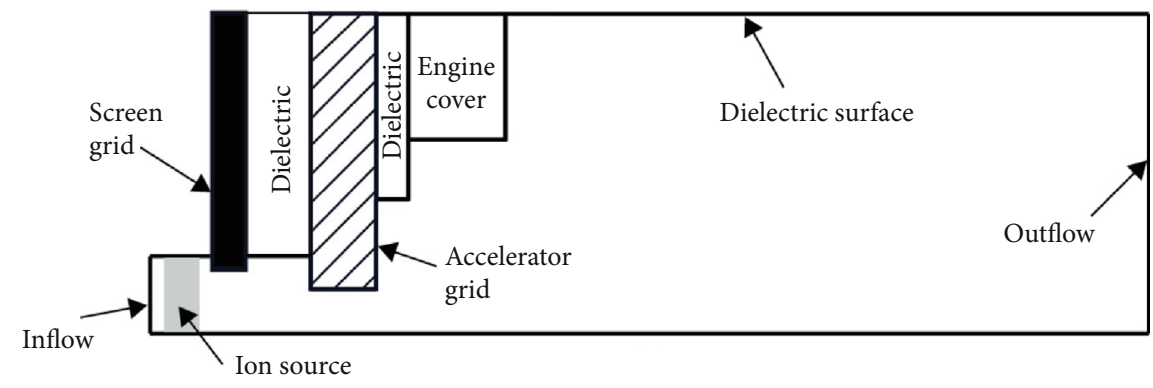

(b)

FIgURE 2: Computational domains with (b) and without (a) metal engine cover.

TABLE 1: Grid parameters used in the simulation.

\begin{tabular}{lc}
\hline Parameter & Value \\
\hline Screen grid diameter $(\mathrm{mm})$ & 2 \\
Accelerator grid diameter $(\mathrm{mm})$ & 1.4 \\
Screen grid thickness $(\mathrm{mm})$ & 0.5 \\
Accelerator grid thickness $(\mathrm{mm})$ & 0.5 \\
Distance between two grid holes $(\mathrm{mm})$ & 1 \\
\hline
\end{tabular}

\section{Results and Discussion}

4.1. Radial Electric Field and Divergence Angle. Figure 3 shows the velocity distribution of $\mathrm{Xe}^{+}$ions obtained from PIC simulation which explains the envelop of the ion beam trajectories. The upper image corresponds to the case without the metal engine cover, and the lower image corresponds to the case when the engine cover is installed. It can be seen in the figure that the grid geometries are well designed such that the ion beam exhibits very directional trajectories without hitting the accelerator grid which infers a good ion perveance. The maximum ion velocity reaches up to $6 \times 10^{4} \mathrm{~m} / \mathrm{s}$ as it passes through the accelerator grid. The divergence angle can be calculated by measuring the beam width at the exit plane. Yellow bars placed on the right exit plane which have the same height indicate that the divergence angle is bigger when the engine cover is installed.

Divergent motion of the ions outside the grid system is linearly dependent on the radial electric field. Figure 4 shows the electric field distributions in radial direction at $z=0.5$ $\mathrm{mm}$ away from the outer surface of the accelerator grid. It can be seen that the radial electric field is stronger when the engine cover is installed. Larger radial electric field enhances the ion motion in the radial direction away from the centerline, which results in more diverged ion trajectory as shown in Figure 3(b). The increment in the radial electric field strength ranges from $15 \%$ at the centerline to $19 \%$ at $r=0.7 \mathrm{~mm}$ away from the centerline.

The accelerator grid voltage modifies not only the velocity of ions emitted through the grid system but also modifies the radial electric field. Figure 5 shows the divergence angle of the ion beam for various accelerator grid voltages. As the negative potential on the accelerator grid increases, the total voltage drop across the grid system increases. However, the beam current increases simultaneously, and hence, the perveance increases. When the perveance value is less than the optimal value, the beam divergence angle is inversely proportional to the perveance [12]. As shown in Figure 5, the divergence angle increases as the accelerator grid voltage increases due to a reduced beam current. However, the effect of the engine cover becomes weaker due to a weaker radial electric field. It is interesting to notice from the computational result that the divergence angle slightly drops at $-325 \mathrm{~V}$ when the engine cover is installed, which is not observed when the engine cover is removed.

4.2. Ion Beam Distribution from Faraday Probe. The effect of the engine cover is experimentally investigated by Faraday probe measurement. Figure 6 shows the schematic drawing of the Faraday probe measurement system. The ion engine developed in our laboratory is $50 \mathrm{~W}$ class RF ion thruster with beam diameter on the accelerator grid is $45 \mathrm{~mm}$. Surface area of the collector of Faraday probe from which the beam current is measured is $10 \mathrm{~mm}$. The ion engine is placed at a specific location from the probe and the probe translates in $r$-direction as shown in the figure. Distribution of the ion beam current informs about the divergence angle [20]. Beam 


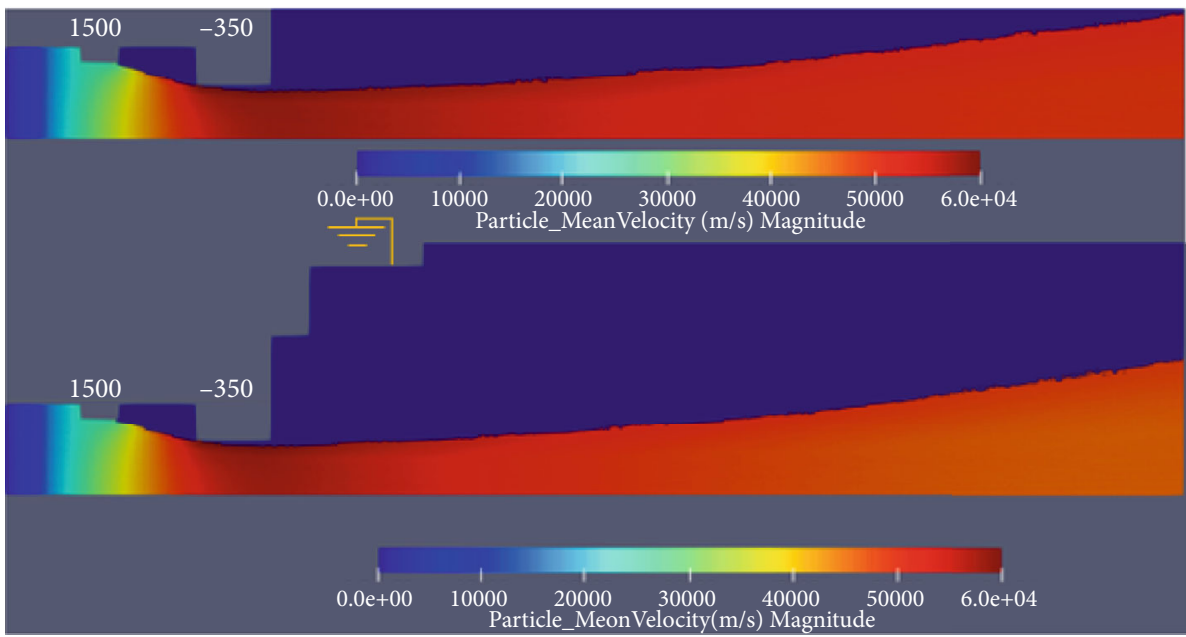

FIGURE 3: Velocity distribution of $\mathrm{Xe}^{+}$with (lower) and without (upper) the engine cover.

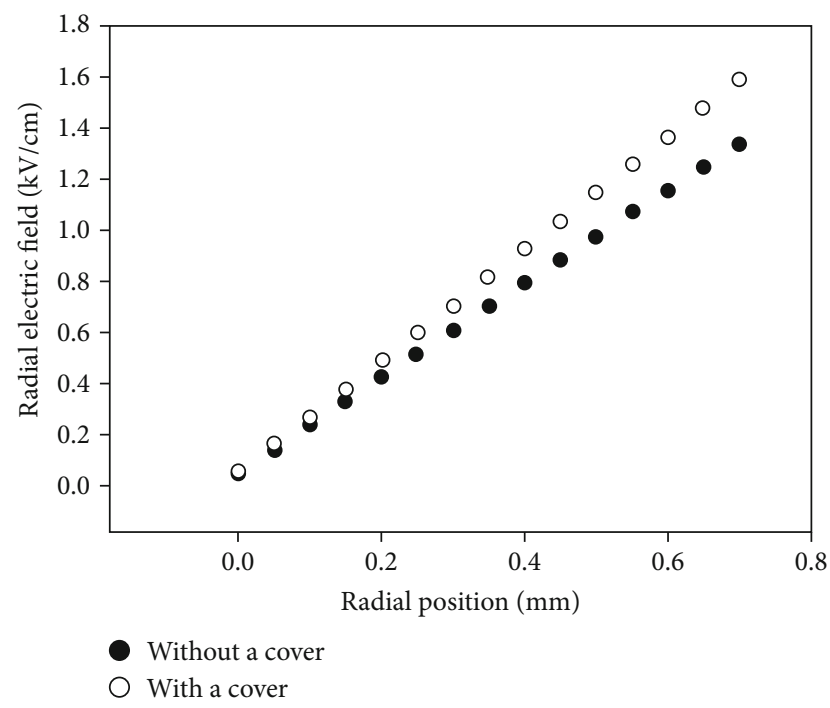

Figure 4: Variation of radial electric field with and without the engine cover.

current density $\left(J_{B}\right)$ is calculated by

$$
J_{B}=\sum_{k} e \Gamma_{k} Z_{k}=\frac{I_{E P}}{A_{C}+k_{G}} k_{S E E}
$$

where $I_{E P}$ is measured beam current, $A_{c}$ is the area of the collector surface of the probe, $k_{G}$ is a correction for the collector area, and $k_{S E E}$ is a correction factor accounting for the secondary emission on the collector [21]. $k_{G}$ and $k_{S E E}$ are calculated by

$$
k_{G}=\pi\left(R_{G R}^{2}-R_{C}^{2}\right)\left(\frac{2 \pi R_{C} h_{C}}{2 \pi R_{C} h_{C}+2 \pi R_{G R} h_{G R}}\right) \text {, }
$$

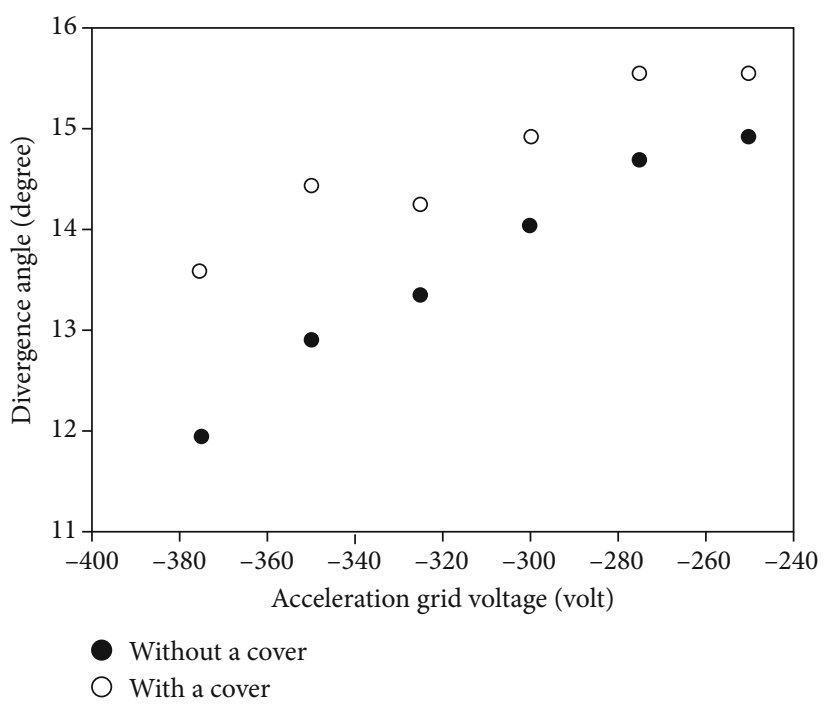

FIGURE 5: Divergence angle for various accelerator grid voltages.

and

$$
k_{S E E}=\frac{1}{1+\Sigma_{k}\left(\Omega_{k} \gamma_{k} / Z_{k}\right)},
$$

where $R_{C}$ is the radius of the collector, $h_{C}$ is the height of the collector, $R_{G R}$ is the radius of the guard ring, $h_{G R}$ is the height of the guard ring, and $\Omega_{k}, Z_{k}, \gamma_{k}$ are ion current fraction, charge number, and secondary emission yield, respectively. The maximum uncertainty level of the current measurement system which was obtained at $5 \mathrm{sccm}$ and $55 \mathrm{~W}$ is about $0.5 \mathrm{~mA} / \mathrm{cm}^{2}$ for a $95 \%$ confidence interval calculated by assuming that the statistical error follows the student's $T$ distribution used when the number of samples is not large [22].

Figure 7 shows the distribution of the current density of the ion beam measured at $z=15 \mathrm{~cm}$ away from the accelerator grid surface. The RF input power is $50 \mathrm{~W}$, and the mass 


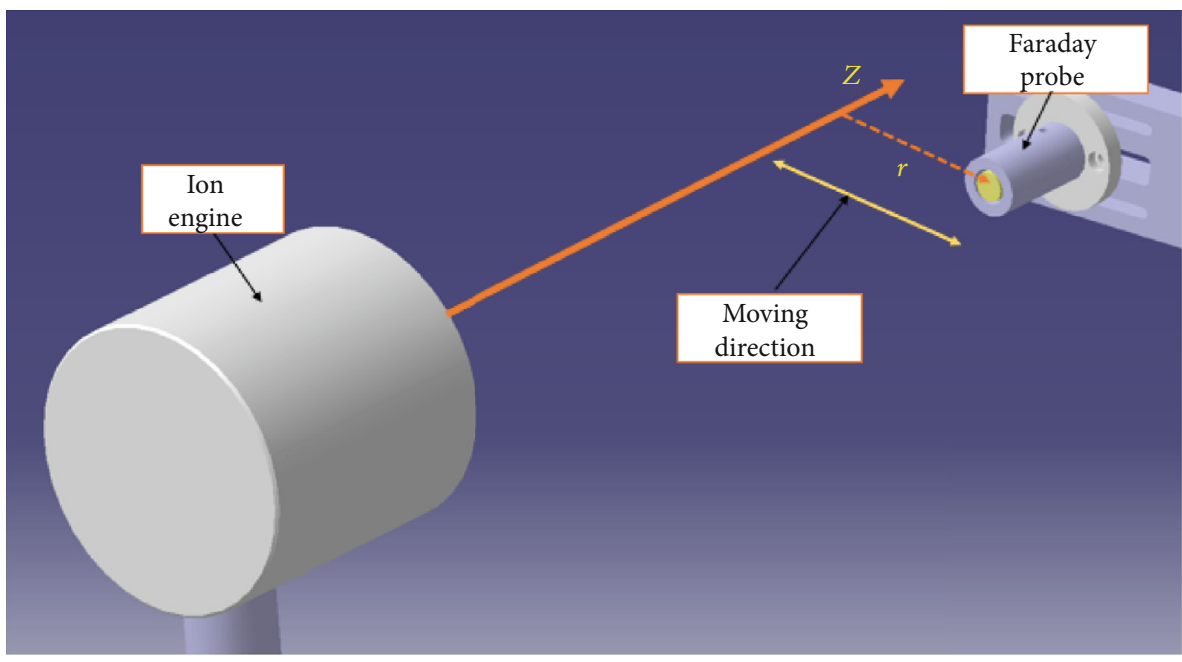

FIGURE 6: Schematic drawing of Faraday probe measurement system.

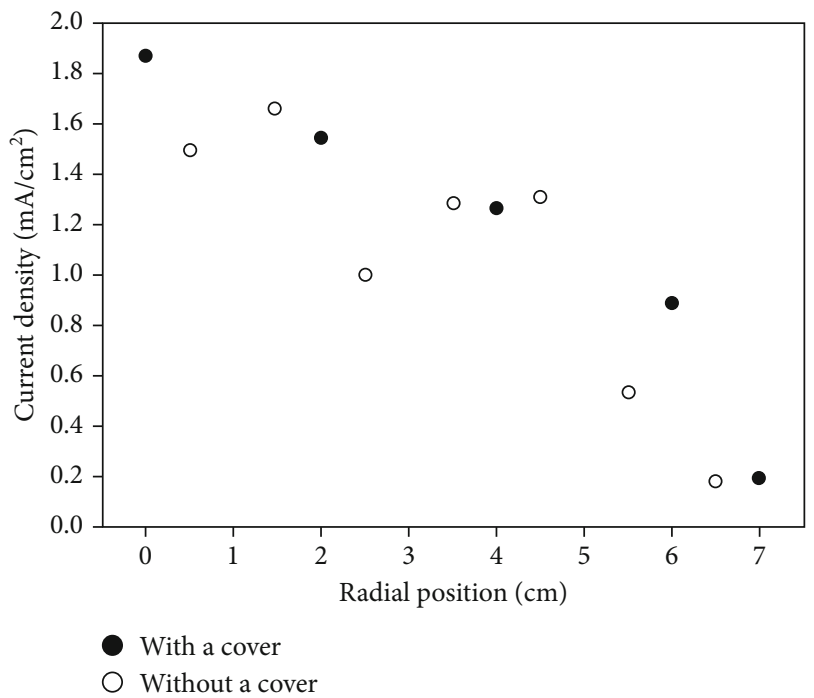

FIgURE 7: Distribution of current density in the radial position when the engine cover is in use (dot symbol) and when the engine cover is removed (hollow circular symbol).

flow rate is $3 \mathrm{sccm}$. The dot symbol corresponds to the case with the engine cover, and the hollow circular symbol corresponds to the case without the engine cover. It can be seen that the current density in both cases drops monotonically in the radial direction. Considering the uncertainty level, it is marginal to state that the divergence angle of the ion beam is larger when the engine cover is installed which is consistent with the PIC simulation result.

Figure 8 shows the current density distribution of the ion beam for various RF powers when the engine cover is installed. Mass flow rate is set constant at $4 \mathrm{sccm}$, and the probe is placed at $10 \mathrm{~cm}$ away from the accelerator grid surface. As expected, the beam current density is highest along the centerline and drops as the Faraday probe moves in the radial direction. As RF power increases, the beam current density increases across the entire radial positions. However,

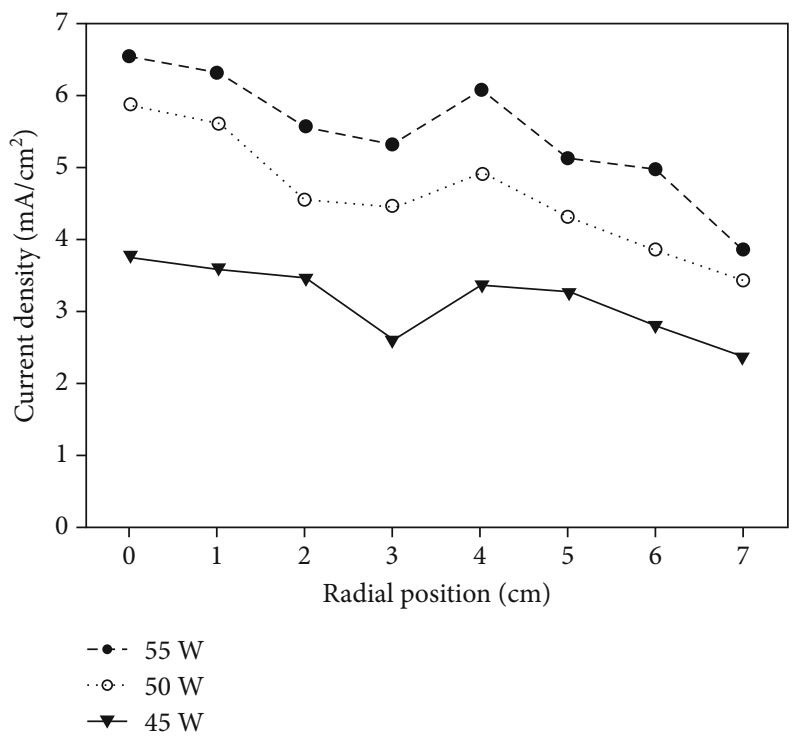

FIGURE 8: Current density distribution in radial position for various RF powers.

it is observed that the current density increases again as the probe moves from $3 \mathrm{~cm}$ to $4 \mathrm{~cm}$ for each input power. Figure 9 shows the current density distribution of the ion beam for different mass flow rates. The RF input power is $50 \mathrm{~W}$, and the probe is placed at $10 \mathrm{~cm}$ away from the accelerator grid surface. As the mass flow rate increase, the beam current density increases across the entire radial positions. However, it is also observed that the current density rises again at $4 \mathrm{~cm}$ as observed in Figure 8. It is conjectured that the electric field which is mainly responsible for the particle motion under very weak induced magnetic field does not change monotonically in the radial direction. Another reason can be a nonuniform density distribution inside the discharge chamber due to a conical shape of the ion thruster. As the flow rate decreases, the 2 nd peak becomes relatively 


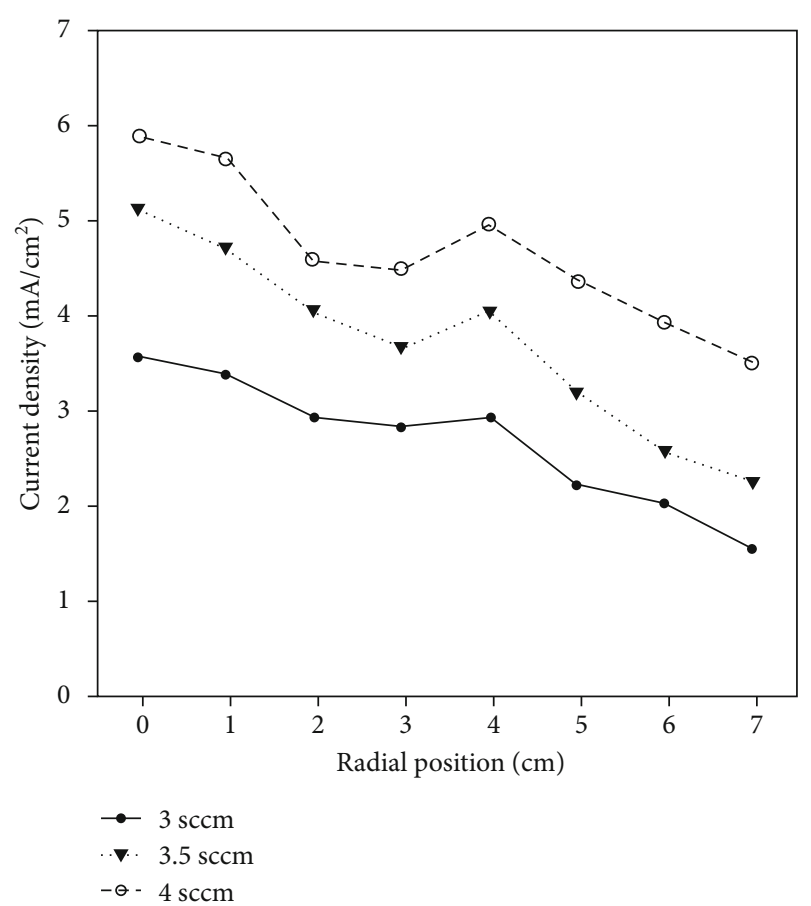

FIGURE 9: Current density distribution in radial position for various flow rate.

weak. Full characterization of a RF ion thruster requires a thrust measurement which is currently unavailable.

\section{Conclusions}

PIC simulation result has shown that the radial electric field is increased by up to $19 \%$ when the metal engine cover is installed. This explains an increase in the divergence angle of the ion beam by $10.52 \%$ due to the engine cover. The divergence angle which is inversely proportional to the ion perveance increases as the accelerator grid voltage increases because of the lower beam current resulting in the lower ion perveance. Faraday probe measurement shows similar behavior where the current density drops off earlier in the radial direction when the engine cover is installed. Beam current density increases as the input RF power increases and the mass flow rate increases. However, it is observed that there exists the second peak in the current density at $r=4$ $\mathrm{cm}$ which is repeatedly observed in the tested operating conditions for $50 \mathrm{~W}$ class $\mathrm{RF}$ ion thruster.

\section{Data Availability}

The data of the results of this study is related to the development of $50 \mathrm{~W}$ class RF ion thruster. Upon request, a part of the data can be obtained from the corresponding author.

\section{Conflicts of Interest}

The authors declare that there are no conflicts of interest regarding the publication of this paper.

\section{Acknowledgments}

This paper is supported by the 2018 Research Fund of the University of Ulsan.

\section{References}

[1] E. Y. Choueiri, "A critical history of electric propulsion: the first 50 years (1906-1956)," Journal of Propulsion and Power, vol. 20, no. 2, pp. 193-203, 2004.

[2] D. Lev et al., The Technological and Commercial Expansion of Electric Propulsion in the Past 24 Years, the 35th International Electric Propulsion Conference, Atlanta, Georgia, USA, 2017.

[3] S. Mazouffre, "Electric propulsion for satellites and spacecraft: established technologies and novel approaches," Plasma Sources Science and Technology, vol. 25, no. 3, p. 033002, 2016.

[4] R. G. Jahn and E. Y. Choueiri, "Electric propulsion," Encyclopedia of Physical Science and Technology, vol. 5, 2002.

[5] M. Tsay, K. Hohman, and L. Olson, "Micro RF ion engine for small satellite applications," in the 23rd Annual AIAA/USU Conference on Small Satellite, Logan, Utah, 2009.

[6] M. Martinez-Sanchez and J. E. Pollard, "Spacecraft electric propulsion-an overview," Journal of Propulsion and Power, vol. 14 , no. 5, pp. 688-699, 1998.

[7] B. Dankongkakula and R. E. Wirz, "Miniature ion thruster ring-cusp discharge performance and behavior," Journal of Applied Physics, vol. 122, Article ID 243303, 2017.

[8] D. L. Lockwood and V. Hamza, "Space-charge-flow theory and electrode design for electrostatic rocket engines," NASA, vol. D-1461, 1962.

[9] J. Hyman Jr., W. O. Eckhardt, R. C. Knechtli, and C. R. Buckey, "Formation of ion beams from plasma sources. I," AIAA Journal, vol. 2, no. 10, pp. 1739-1748, 1964.

[10] W. C. Laihem, "Ion accelerator designs for Kaufman thrusters," Journal of Spacecraft and Rockets, vol. 6, no. 11, pp. 1237-1242, 1969.

[11] G. Aston, "“Ion optics study", 15-cm mercury ion thruster research,” NASA, vol. CR-134905, pp. 49-70, 1975.

[12] G. Aston, H. R. Kaufman, and P. J. Wilbur, "Ion beam divergence characteristics of two-grid accelerator systems," AIAA Journal, vol. 16, no. 5, pp. 516-524, 1978.

[13] J. R. Coupland, T. S. Green, D. P. Hammond, and A. C. Riviere, "A study of the ion beam intensity and divergence obtained from a single aperture three electrode extraction system," Review of Scientific Instruments, vol. 44, no. 9, pp. 12581270, 1973.

[14] J. R. Pai and N. Venkatramani, "Beam divergence and ion density measurements for an induction coupled ion beam source," Review of Scientific Instruments, vol. 63, no. 11, pp. 5234-5236, 1992.

[15] D. M. Goebel, "Analytical discharge model for RF ion thrusters," IEEE Transactions on Plasma Science, vol. 36, no. 5, pp. 2111-2121, 2008.

[16] Esgee Technologies, "Glow/for Viz Glow," 2018, http:// esgeetech.com/products/vizglow-plasma-modeling/Viz.

[17] P. T. D. Quang and J. Shin, "Better prediction of the performance of a radio-frequency ion thruster," Journal of the Korean Physical Society, vol. 76, no. 2, pp. 137-144, 2020.

[18] E. Turkoz, F. Sik, and M. Celik, "A study of ion thruster optics through particle simulations and evaluation of the near plume plasma properties," the 50th Joint Propulsion Conference, 2014. 
[19] C. K. Birdsall, "Particle-in-cell charged-particle simulations, plus Monte Carlo collisions with neutral atoms, pic-mcc," IEEE Transactions on Plasma Science, vol. 19, no. 2, pp. 65$85,1991$.

[20] W. Huang, R. Shastry, D. A. Herman, G. C. Soulas, and H. Kamhawi, "A new method for analyzing near-field Faraday probe data in hall thrusters," in the 49th AIAA/ASME/SAE/ASEE Joint Propulsion Conference, AIAA, pp. 2013-4118, San Jose, CA, 2013.

[21] D. L. Brown, M. L. R. Walker, J. Szabo, W. Huang, and J. E. Foster, "Recommended practice for use of Faraday probes in electric propulsion testing," Journal of Propulsion and Power, vol. 33, no. 3, pp. 582-613, 2017.

[22] R. V. Hogg, J. McKean, and A. T. Craig, Introduction to Mathematical Statics, Pearson Education, 2005. 